\title{
Erstnachweis der Kerbameise Formica pressilabris Nylander 1846 (Hymenoptera: Formicidae) in Schleswig-Holstein
}

\author{
First record of Formica pressilabris Nylander 1846 (Hymenoptera: \\ Formicidae) from Schleswig-Holstein
}

\author{
Uwe Sörensen \\ Süderlügum, usoerensen54@web.de
}

\author{
Schlüsselwörter: \\ Süderlügumer Binnendünen, \\ Formica pressilabris, F. forsslundi, \\ F. exsecta, F. uralensis, F. picea, \\ Morphologie, Bionomie
}

\section{Keywords:}

Süderlügumer Binnendünen, Formica pressilabris, F. forsslundi, F. exsecta, F. uralensis, F. picea, morphology, bionomy

\begin{abstract}
Zusammenfassung
Es wird das Vorkommen der Kerbameise Formica pressilabris im Naturschutzgebiet Süderlügumer Binnendünen im Nordwesten Schleswig-Holsteins dargestellt mit einigen Daten zur Bionomie der Art sowie zur Struktur des Biotops. Der Fund wird im Rahmen der Gesamtverbreitung der Art diskutiert. Es handelt sich um den Erstnachweis für Schleswig-Holstein.
\end{abstract}

\begin{abstract}
The ant Formica pressilabris is recorded in the nature reserve Süderlügumer inland dunes in the northwest of Schleswig-Holstein. Data on the bionomy of the species and the structure of its biotope is presented. The record is discussed in the context of the overall distribution of the species. This is the first record of this species in the federal state of Schleswig-Holstein.
\end{abstract}

\section{Einleitung}

Die Kerbameisen bilden die Untergattung Coptoformica innerhalb der Gattung Formica, zu denen die allgemein bekannten Waldameisen (Formica sensu stricto) gehören. Die Kerbameisen zeichnen sich durch eine Einbuchtung im Hinterhaupt aus, die mit bloßem Auge als Einkerbung erkennbar ist.

Im nordwestlichsten Binnendünengebiet SchleswigHolsteins, im Raum Süderlügum konnte bereits 1986 erstmals für Deutschland ein Vorkommen der Kerbameise Formica forsslundi nachgewiesen werden (Sörensen 1999). Bei einer Bestandserhebung im Naturschutzgebiet Süderlügumer Binnendünen im Jahre 2017 im Auftrage der Schleswig-Holsteinischen Landesforsten (SHLF) wurde nun neben dem zusätzlichen Auftreten mehrerer Nester von Formica exsecta mit Formica pressilabris eine weitere seltene Kerbameisenart in diesem Gebiet entdeckt.
In dieser Arbeit werden der Standort, das bisher bekannte regionale Vorkommen und einige Daten zur Bionomie der Art vorgestellt, um einerseits diesen für die Ameisenfauna Deutschlands wichtigen Fund $\mathrm{zu}$ publizieren und andererseits die Bedeutung des Naturschutzgebietes Süderlügumer Binnendünen für die Ameisenfauna und den Artenschutz zu verdeutlichen.

\section{Die Untergattung Coptoformica}

Das äußerlich sofort auffallende, aber nicht auf die Untergattung Coptoformica beschränkte, Merkmal ist die Einkerbung des Hinterhauptes. Dabei handelt es sich aber nicht um eine Kerbe im eigentlichen Sinne, sondern um eine Erweiterung der Hinterhauptsecken, in der die verlängerten Mandibelmuskeln inserieren und für eine besonders kräftige Beißfähigkeit verantwortlich sind (nach Dlussky 1964 und Dietrich 1998, vgl. Agosti 1989, 


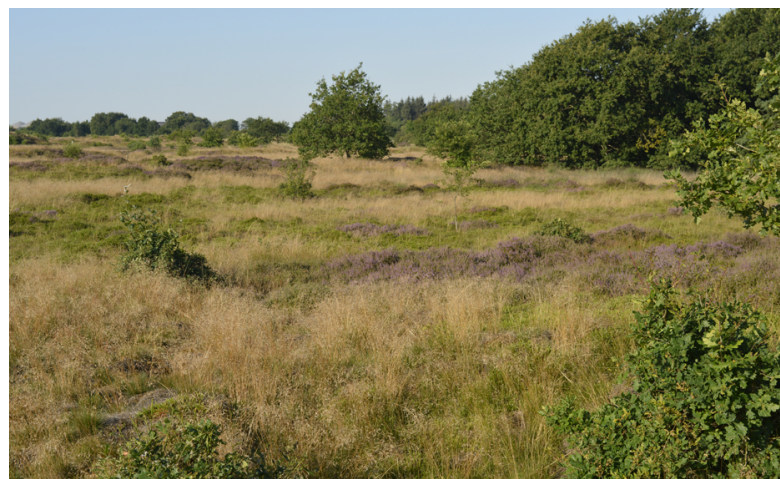

Abb. 1: Biotop des Nestes 1 von Formica pressilabris am Rande eines Eichengehölzes. Die Fläche besteht aus einem kleinräumigen Muster von Beständen der Besenheide (Calluna vulgaris), Krähenbeere (Empetrum nigrum) und Geschlängelter Schmiele (Deschampsia flexuosa). Es ist auch junger Aufwuchs der Stieleiche (Quercus robur) und der Sandbirke (Betula verrucosa) erkennbar. Foto: U. Sörensen, 7.8.2020.

S. 32 ff., Seifert 2000, S. 525). Mit Hilfe der Mandibeln zerlegen die Arbeiterinnen Pflanzenmaterial, um damit einen Nesthügel zu errichten. Außerdem können sie sich mit Hilfe des kräftigen Bisses gut gegen feindliche Ameisen und andere Prädatoren verteidigen.

Nach Seifert $(2000,2018)$ gibt es in Mittel- und Nordeuropa 7 Arten dieser Untergattung: Formica bruni, F. exsecta, $F$. fennica, $F$. foreli, $F$. forsslundi, $F$. pressilabris und F. suecica. Außer F. fennica und F. suecica, die nur in Skandinavien, Finnland, Osteuropa und in alpinen Regionen vorkommen, sind alle Arten in Deutschland nachgewiesen. Die Arten F. foreli und F. pressilabris sind äußerlich sehr ähnlich und können als Schwesterarten bezeichnet werden (Seifert 2007). Das Areal von F. foreli liegt westpaläarktisch und südlicher, das von $F$. pressilabris gesamtpaläarktisch und nördlicher (subboreal) (vgl. Schultz \& Seifert 2007).

Die Determination der Arten erfolgte hauptsächlich nach den Bestimmungsschlüsseln von Seifert (2007, 2018). Ergänzend herangezogen wurde auch der Bestimmungsschlüssel von Czechowski et al. (2012). Dr. Bernhard Seifert, Senckenberg Museum für Naturkunde, Görlitz, überprüfte und bestätigte die Determination der Art Formica pressilabris.

\section{Die Differenzialmerkmale von Formica pressilabris}

Körpermerkmale der Arbeiterinnen: Größe 4,5-6,5 mm lang, eingekerbter Hinterhauptsrand, Farbe rötlich mit dunklem Bereich auf dem Kopf und dem Prothorax. Der

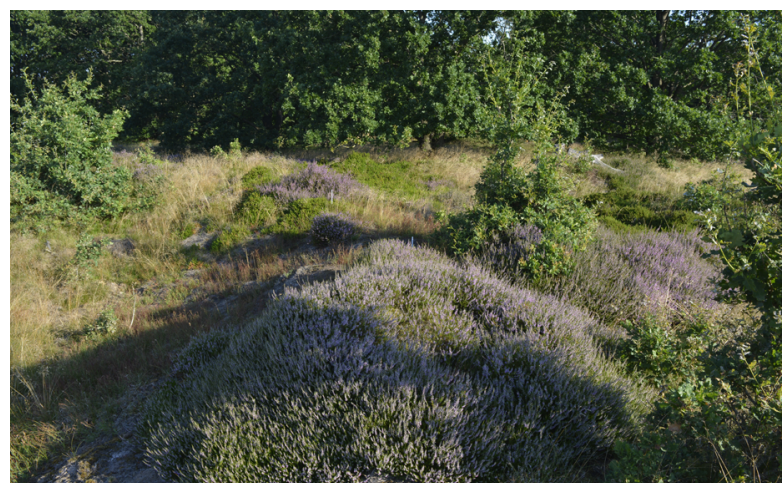

Abb. 2: Standort des Nestes 1 in $10 \mathrm{~m}$ Entfernung des Eichengehölzes. Im Vordergrund ist ein kräftiger Calluna-Bewuchs auf einem länglichen Dünenzug erkennbar. Direkt dahinter befindet sich im Halbschatten das Nest 1, das sich nach links in eine längliche Vertiefung absenkt. Foto: U. Sörensen, 7.8.2020, 8:13 Uhr MEZ.

ganze Körper weist eine extrem geringe, abstehende Behaarung (Setae) auf. Auch die anliegende Behaarung (Pubeszens) ist sehr lückig, sodass die Tiere bei oberflächlicher Betrachtung etwas glänzend wirken. Im Schwedischen wird die Art folglich als ,blank hedmyra bezeichnet (Douwes et al. 2012). Auch die Augen zeigen keine oder nur eine bei größerer Vergrößerung sehr geringe Behaarung auf. Von F. forsslundi lässt sie sich dadurch unterscheiden, dass ihr Hinterleib (Gaster) erst ab dem 3. oder 4. Segment abstehende Haare aufweist, F. forsslundi bereits ab dem 1 . oder 2. Segment. Von F. foreli (,matt hedmyra') lässt sie sich durch die deutlich lückigere Behaarung im Ocellen-Dreieck auf der Stirn und auf dem gesamten Körper trennen.

\section{Bemerkungen zur Lebensweise von F. pressilabris}

Die flachen Nesthügel bestehen zumeist aus zerbissenen Grasstückchen, häufig mit Erdmaterial durchmischt. In Skandinavien tritt sie in Einzelnestern oder in kleinen Ansammlungen auf, in Polen sind große Nestkolonien von über 100 Einzelnestern bekannt (Czechowski 1975, Czechowski et al. 2012). Die größte bekannte Kolonie in Nordwesteuropa besteht seit längerem an der Küste der Niederlande mit ca. 150 Nesthügeln auf einer Fläche von 1 qkm (Boer 2010). Die Art zeigt eine starke Tendenz zu offenem, trockenem Grasland oder Waldrändern. Die Nestgründung erfolgt sozialparasitisch bei verschiedenen Arten der Serviformica-Gruppe. 


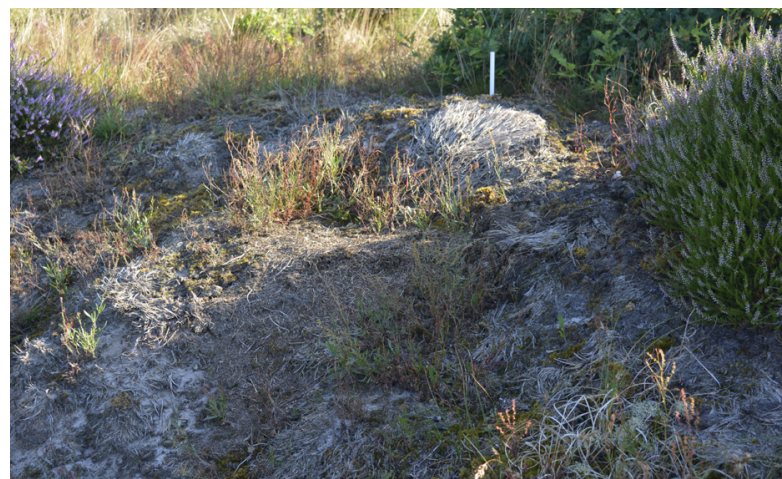

Abb. 3: Die Oberfläche von Nest 1. Das Nest liegt an einem Sandhang und ist nach Südwesten exponiert. Die Kuppe ist sehr flach, besteht hauptsächlich aus zerbissenen Halmen von Deschampsia flexuosa. Umgeben wird die Nestkuppe von Rumex acetosella-Pflanzen. Zum Zeitpunkt der Aufnahme liegt sie noch im Halbschatten des östlich angrenzenden Calluna vulgaris-Bewuchses. Foto: U. Sörensen, 7.8.2020, 8:17 Uhr MEZ.

Als Nahrung werden die Ausscheidungen von Wurzelläusen an den Wurzeln der Gräser und auch an oberirdischen Pflanzenorganen aufgenommen. Daneben werden auch in geringerem Maße Kleintiere erbeutet oder Aas als Nahrung angenommen.

\section{Verbreitung und Gefährdung von F. pressilabris}

Das Verbreitungsgebiet dieser Art liegt im gemäßigten bis südborealen Klimagebiet der Paläarktis Eurasiens, von der Tiefebene bis in den submontanen Bereich mit mehr kontinentaler Ausprägung (Seifert 2000, 2018). In Mitteleuropa kommt sie in Dänemark und Südskandinavien (Collingwood 1979, Nielsen \& Larsen 2012, Douwes et al. 2012, Ødegaard et al. 2015), den Niederlanden (Loon 2004, Boer 2010), dem Alpenraum (Kutter 1956, 1977, Glaser \& Müller 2003), Polen (Czechowski et al. 2012) und Russland (Seifert 2018) vor. In Deutschland ist sie aktuell bisher nur mit zwei kleineren Vorkommen aus dem Raum Eberswalde (Brandenburg) und dem südöstlichen Mecklenburg-Vorpommern bekannt (Wesenigk-Sturm 2002, Seifert 2007). Aus Süderlügum stammt jetzt der dritte rezente Nachweis für Deutschland. Ein bayerisches und ein Vorkommen aus dem deutsch-belgischen Grenzgebiet sowie ein älteres (1910) aus dem Hamburger Raum scheinen erloschen zu sein (Sonnenburg 2005, Schultz \& Seifert 2007).

Der Gefährdungsstatus von F. pressilabris nach der Roten Liste der Ameisen Deutschlands (Seifert 2011) ist als Kategorie 1, vom Aussterben bedroht, eingeordnet.

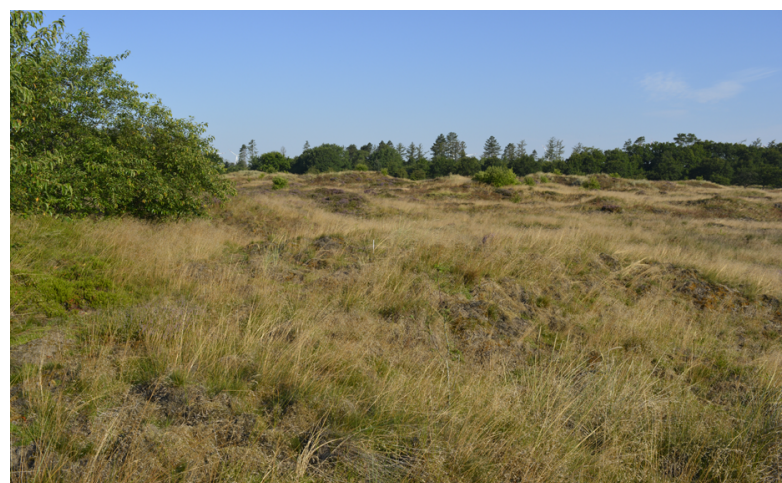

Abb. 4: Biotop des Nestes 2 von Formica pressilabris. Das Nest liegt in der Mitte des Bildes auf der Kuppe eines Dünenzuges, der hauptsächlich von Deschampsia flexuosa bewachsen ist. Am linken Bildrand ist ein Gebüsch von Prunus serotina sowie eine Empetrum nigrum-Fläche zu sehen. Foto: U. Sörensen, 7.8.2020, 8:30 Uhr MEZ.

\section{Material und Methoden}

Seit 1986 wird das NSG Süderlügumer Binnendünen vom Autor näher untersucht. Begleitend zu den Schutzmaßnahmen zur Heideregeneration wurden mehrere Gutachten und Stellungnahmen für die Naturschutzbehörden angefertigt (u.a. Sörensen 1993, 1996, 2006). Im Jahre 2017 wurde für die Schleswig-Holsteinischen Landesforsten als betreuende Institution für das Naturschutzgebiet eine Erhebung zur Verteilung und zum Bestand der seltenen Ameisenarten erarbeitet (Sörensen 2017). Das durch das unregelmäßige Relief schwer überschaubare Gebiet wurde dafür mittels Fluchtstangen in Einzelflächen unterteilt und dann fast vollständig nach Nesthügeln abgesucht. Die in der Vegetation verborgenen jungen Nestgründungen sind dabei allerdings nicht oder nur zufällig auffindbar, da die Nester zuerst nur aus den unterirdischen bzw. innerhalb der Grasnarbe befindlichen Nestgängen und Nesthöhlen bestehen.

\section{Das Untersuchungsgebiet Süderlügumer Binnendünen}

Das Untersuchungsgebiet liegt im Nordwesten Schleswig-Holsteins etwa $4 \mathrm{~km}$ südlich der dänischen Grenze (vgl. Sörensen 1999, 2004). Geologisch betrachtet handelt es sich bei diesem Landschaftsausschnitt im Bereich Süderlügum um eine flache Altmoränenkuppe des Lecker Geestkerns, die randlich von spätglazialen und postglazialen Flugsandakkumulationen des Weichselglazials überdeckt wurde. Aus diesen entstanden, 


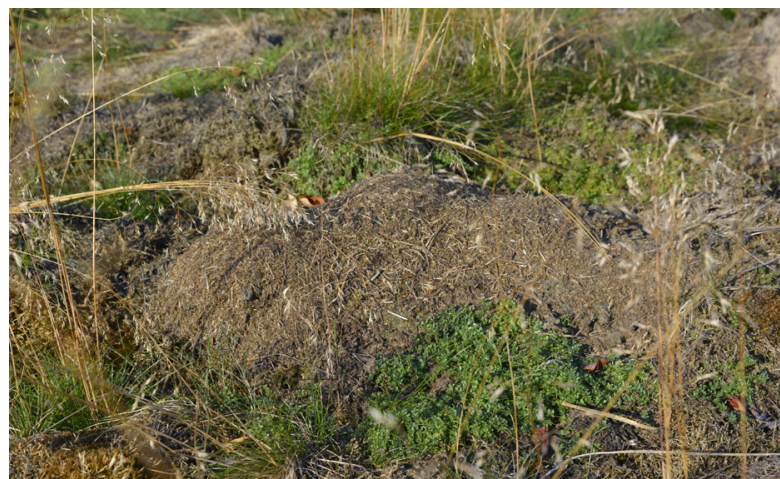

Abb. 5: Nestkuppe von Nest 2. Der Nesthügel ist etwas länglich gebogen und weist vier kleinere Erhebungen auf, unter denen sich Nestkammern befinden. Im Vordergrund ist der Bewuchs von Galium saxatile und Deschampsia flexuosa zu erkennen. Foto: U. Sörensen, 7.8.2020, 8:29 Uhr MEZ

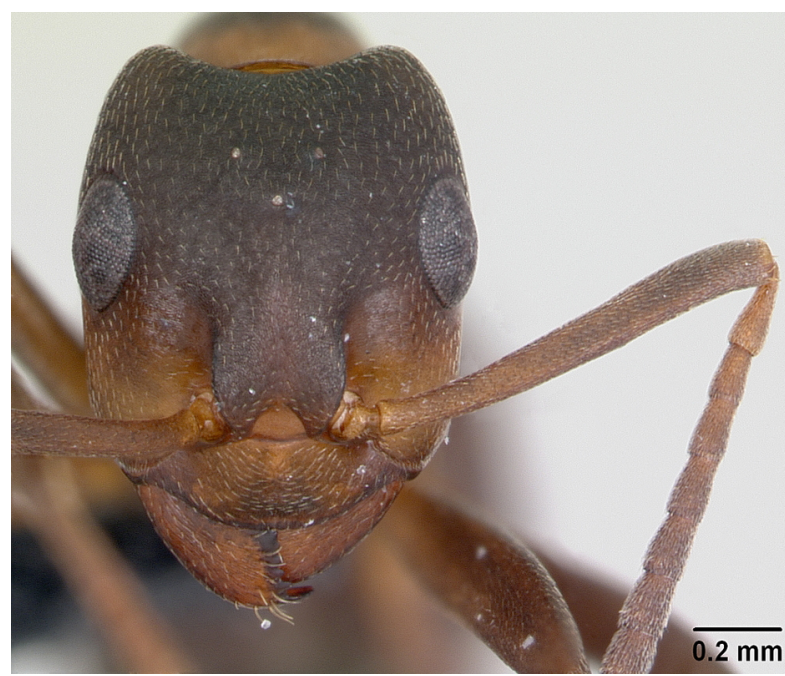

Abb. 7: Kopf von Formica pressilabris in Frontalansicht. Die entscheidenden Merkmale zur Artdiagnose sind das Fehlen der abstehenden Behaarung auf den Augen sowie die große Distanz zwischen den Pubeszenshaaren insbesondere im Ocellen-Dreieck. Foto: April Nobile, Individuum CASENT0173872, mit freundlicher Genehmigung von AntWeb.org, Version 8.41. California Academy of Science, https:// www.antweb.org, letzter Zugriff am 21.8.2020.

wahrscheinlich schon während der letzten Eiszeit, Binnendünenlandschaften. Diese Formation zieht sich in West-Ost-Richtung über ca. 20 km Länge an dem nördlich anschließenden Talsandergebiet der Wiedau-Niederung entlang (Dücker et al. 1957, Woldstedt et al. 1974, Geologischer Dienst 2012).

Das Erscheinungsbild der Dünen ist bereits seit dem Mittelalter erheblich modifiziert worden. Große Teile der ehemaligen Dünenlandschaft sind in Ackerland und Weideflächen umgewandelt, aufgeforstet oder durch Siedlungen überbaut worden (Riedel 1978). An

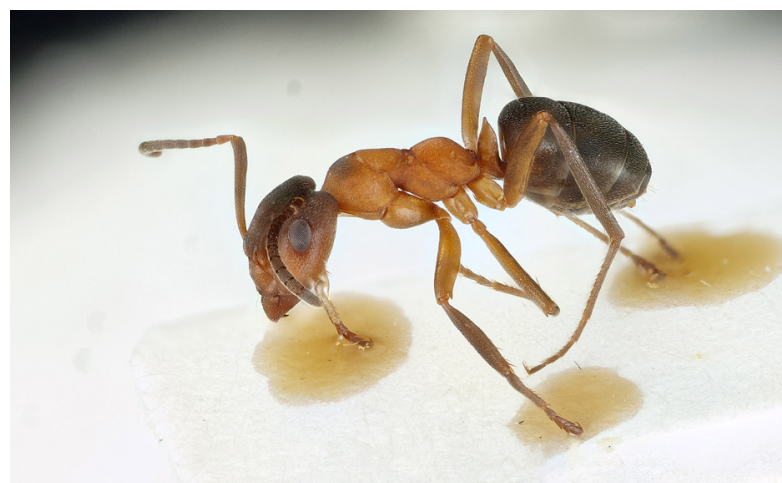

Abb. 6: Arbeiterin von Formica pressilabris in Seitenansicht. Deutlich erkennbar ist die Einkerbung am Hinterhauptsrand sowie die fast völlig fehlende abstehende Behaarung auf der Körperoberfläche. Sie findet sich erst ab dem 3. Gastertergit. Foto: Hajo Hornberg, Flensburg, und Uwe Sörensen, Süderlügum, 26.3.2013.

vielen Abschnitten sind trotzdem auch heute noch Dünenreste $\mathrm{zu}$ entdecken, insbesondere in den Naturschutzgebieten am Rande Süderlügums, NSG Süderlügumer Binnendünen und NSG Süderberge, aber auch in den anschließenden Süderlügumer und Humptruper Forsten, der Feldflur und mitten im Süderlügumer Dorfbereich.

Das Naturschutzgebiet Süderlügumer Binnendünen ist eines der am besten erhaltenen Binnendünengebiete Schleswig-Holsteins und umfasst eine Fläche von ca. 40 ha. Es zeigt ein bewegtes Dünenrelief mit bis zu 8 $\mathrm{m}$ Höhenunterschieden. Bis auf den großen Dünenkessel im östlichen Zentralbereich und den ausgetretenen Pfaden mit Offensandflächen sowie den frischen Plaggflächen der letzten Jahre ist das NSG heute dicht von Heidevegetation bewachsen. Den Hauptteil der Vegetation nehmen Deschampsia flexuosa-Formationen mit geringem Calluna-Anteil ein. Daneben finden sich größere Flächen mit Empetrum nigrum, besonders an den Nordhängen der Dünen, Senken mit Molinia caerulea und Erica tetralix, sowie den Plaggflächen, die größtenteils von Calluna vulgaris neu besiedelt sind.

Plaggmaßnahmen finden zur Heideregeneration seit 1988 regelmäßig im Gebiet statt (van der Ende 1991, 1993, 2008). Die Beweidung durch eine Schafherde wurde seit 2002 mehrmals jeweils nur kurzzeitig im Gebiet ausprobiert, aber wegen der großen Schäden an den Beständen der seltenen Ameisenarten unterbrochen (Sörensen 2006), wird aber seit 2019 kleinräumig wieder fortgesetzt. Die Auswirkungen werden in Zusammenarbeit mit den Mitarbeitern der Landesforsten vom Autor beobachtet und dokumentiert. 


\section{Ergebnisse}

Bei der oben beschriebenen Bestandsaufnahme der seltenen Ameisenarten im NSG Süderlügumer Binnendünen (Sörensen 2017) fielen dem Autor etwas größere, aus zerbissenen Grasstückchen bestehende Nesthügel auf, die ansonsten den Nestern von Formica forsslundi sehr ähnelten. Vier dieser Nester waren von der im Gebiet bisher nicht bekannten $F$. exsecta besiedelt und eines von F. pressilabris. Ca. 1300 Nester konnten durch zahlreiche Stichproben $F$. forsslundi zugeordnet werden.

Im Jahre 2019 bei vorbereitenden Arbeiten für eine Beweidungsmaßnahme im NSG konnte ein weiteres Nest von $F$. pressilabris in ca. $390 \mathrm{~m}$ Entfernung vom ersten Nest gefunden werden.

Das erste Nest hat einen Durchmesser von 50 x $40 \mathrm{~cm}$ und eine Höhe von ca. $10 \mathrm{~cm}$, flach gewölbt und liegt an einem südexponierten Hang. Der Nesthügel besteht aus zerbissenen Grashalmstückchen und größeren Anteilen von Sand und abgestorbenen Pflanzenteilen. In ca. 10 $\mathrm{m}$ Entfernung befindet sich eine mittelgroße Stiel-Eiche (Quercus robur) nahe des nordwestlichen Randes des NSG, an dem sich ein Eichen-Birken-Wald entwickelt hat. Die Vegetation der Nestumgebung ist sehr lückig, ist aber insgesamt als Krähenbeerenheide (GenistoCallunetum empetretosum) einzuordnen (nach Runge 1990, Ellenberg 1996). Neben Calluna vulgaris und Empetrum nigrum treten Deschampsia flexuosa und Nardus stricta flächig auf. Außerdem finden sich in der Nähe des Nestes Bestände von Rumex acetosella, Spergularia moresonii, Carex arenaria sowie verschiedene Laubmoosarten, wie z.B. Hypnum sp. und Campylopus introflexus. Auch ein kleines Gebüsch der Stiel-Eiche befindet sich in $1 \mathrm{~m}$ Entfernung des Nestrandes.

In geringer Entfernung befinden sich Nester von Formica rufa, Formica uralensis und Formica forsslundi.

Das Nest befindet sich nach der Entdeckung im Jahre 2017 unter steter Beobachtung durch den Autor und ist auch aktuell noch stark bevölkert, hat sich aber in der Größe des Nesthügels nicht verändert. Die Oberfläche des Nesthügels wies Ende Mai 2020 Trocknungsrisse auf, die trotz der fortgeschrittenen Jahreszeit noch nicht repariert waren. Erst Ende Juni 2020 wurde die Nestkuppe durch die Arbeiterinnen ausgebessert. Im Sommer 2021 wurde das Nest von dem Volk verlassen. In 3 m Entfernung ist ein neuer kleiner Nesthügel entstanden.

Das zweite Nest hat einen Durchmesser von 50 x $40 \mathrm{~cm}$, eine Höhe von $15 \mathrm{~cm}$ und eine flach gewölbte Nestkup- pe auf der ebenen Hochfläche eines Dünenzuges. Die Nestkuppe ist in zwei höhere Bereiche unterteilt, unter denen sich Nestkammern befinden.

Die dichte Vegetationsdecke der Nestumgebung besteht aus verschiedenen Laubmoosarten (z.B. Hypnum sp., Pleurozium schreberi, Campylopus introflexus, Polytrichum sp., Dicranum spurium) und Gräsern (Deschampsia flexuosa, Ammophila arenaria, Carex arenaria) sowie Calluna vulgaris und Galium saxatile. Insbesondere die Gräser zeigen noch deutliche Schäden durch den Trockensommer 2018. Abgestorbene Grasbulte werden von verschiedenen Laubmoosen und Flechten überwachsen. In einer Entfernung von $19 \mathrm{~m}$ befindet sich ein starkes Nest von Formica exsecta und in diesem Umkreis mehrere Nester von Formica forsslundi und Formica picea.

Während der Beweidung im Herbst 2019 wurde das Nest durch eine Drahthaube vor dem Vertritt geschützt. Das Nest ist auch noch im Jahre 2021 stark belebt und die Arbeiterinnen zeigen eine relativ starke Auslaufaktivität. Die Schutzhaube ist wieder entfernt worden.

\section{Diskussion}

Die Nester von F. pressilabris in den Süderlügumer Binnendünen sind sicherlich als Ausläufer der dänischen Vorkommen zu werten. Die beiden nächstgelegenen Vorkommen in Süddänemark liegen $32 \mathrm{~km}$ nördlich bei Arrild und $40 \mathrm{~km}$ nordwestlich auf der Insel Röm innerhalb der Kommune Tondern (eigene Funde). Weitere Nester werden in Südjütland, in dem viele Heidegebiete und trockene Graslandschaften vorhanden sind, zu finden sein. Sie schließen sich an das über ganz Jütland und Seeland lückige Verbreitungsgebiet der Art in Dänemark an (Collingwood 1979, Nielsen \& Larsen 2012). Nach Süden hin scheint eine größere Lücke im Verbreitungsgebiet zu bestehen. Die Entfernung zu dem Fund von 1910 bei Hamburg (Sonnenburg 2005) beträgt 170 $\mathrm{km}$, zu den rezenten Vorkommen in den Niederlanden (Boer 2010) $290 \mathrm{~km}$ und zu den zwei in Ostdeutschland bekannten Vorkommen (Seifert 2007) ca. $390 \mathrm{~km}$.

Die aktuellen Befunde lassen vermuten, dass auf den im gesamten Norddeutschland vorhandenen Vorzugshabitaten der Art, mehr oder weniger trockenes, offenes Grasland und geschützte Waldrandlagen, weitere Nester von Formica pressilabris zu finden sein müssten. Auch unter der Vielzahl von Coptoformica-Nestern im Naturschutzgebiet Süderlügumer Binnendünen sind noch weitere Nester dieser Art zu erwarten. 


\section{Danksagung}

Ich danke Herrn Dr. Bernhard Seifert, Senckenberg Museum für Naturkunde, Görlitz, für die Überprüfung und Bestätigung der Determination der Art Formica pressilabris. Außerdem danke ich Dipl.-Biol. Holger Sonnenburg, Blomberg, Dr. Michael Kuhlmann, Kiel, und Dipl. Geogr. Jörn Krütgen, Kiel, für die Durchsicht des Manuskriptes und wertvolle Hinweise.

\section{Autor}

\section{Uwe Sörensen}

Uwe Sörensen ist Oberstudienrat a.D. Nach seinem Studium der Biologie und Geographie an der Universität Hannover unterrichtete er 38 Jahre an Gymnasien in Schleswig-Holstein. Er promovierte in Zoologie mit einer Arbeit zum Thema Ökologie der Kerbameise Formica forsslundi. Sein Arbeitsgebiet ist die regionale Fauna und auch Flora im nordwestlichen Schleswig-Holstein und dem angrenzenden Dänemark. Insbesondere werden die aculeaten Hymenopteren erfasst mit dem Schwerpunkt Ameisen.

\section{Literaturverzeichnis}

Agosti D (1989) Versuch einer phylogenetischen Wertung der Merkmale der Formicini (Hymenoptera, Formicidae), Revision der Formica exsecta-Gruppe und Liste der Formicidae Europas. Dissertation, Eidgenössische Technische Hochschule Zürich, doi: 10.3929/ethza-000510347.

Boer P (2010) Mieren van de Benelux. Stichting Jeugdbondsuitgeverij, s'Graveland.

Collingwood CA (1979) The Formicidae (Hymenoptera) of Fennoscandia and Denmark. Fauna Entomologica Scandinavica Vol. 8. Scandinavian Science Press, Klampenborg

Czechowski W (1975) Bionomics of Formica (Coptoformica) pressilabris Nyl. (Hymenoptera, Formicidae). Ann. Zool. 33 (8):103-125.

Czechowski W, Radchenko A, Czechowska W, Vepsäläinen K (2012) The Ants of Poland with reference to the myrmecofauna of Europe. Natura optima dux Foundation, Warszawa.

Dietrich CO (1998) Plünderung eines Formica lemani-Volkes durch Formica exsecta (Hymenoptera: Formicidae) am Göller (Österreich: Niederösterreich) mit einer funktionellen Deutung des Beißverhaltens der Formica exsecta-Gruppe. Myrmecologische Nachrichten Bürs 2:19-34.

Dlussky GM (1964) The Ants of the Subgenus Coptoformica of the Genus Formica (Hymenoptera, Formicidae) of the USSR. Zoologichesky Zhurnal 43:1026-1040.

Douwes P, Abenius J, Cederberg B, Wahlstedt U (2012) Steklar: Myror - getingar. Hymenoptera: Formicidae - Vespidae. ArtDatabanken SLU, Uppsala.

Dücker A, Maarleveld G (1957) Hoch- und spätglaziale äolische Sande in Nordwestdeutschland und in den Niederlanden. Geologisches Jahrbuch 73:215-234.

Ellenberg H (1996) Vegetation Mitteleuropas mit den Alpen in ökologischer Sicht. 5. Aufl. Ulmer, Stuttgart.

Ende Mvd (1990) Das Plaggen - eine Maßnahme der historischen Heidebewirtschaftung. In: Beiträge zu Naturschutz und Landschaftspflege 1987-1991. Landesamt für Naturschutz und Landschaftspflege Schleswig-Holstein, Kiel, S. 143-145.

Ende Mvd (1993) Heidemanagement in Schleswig-Holstein. NNA-Berichte6 (3):53-62.

Ende Mvd (2008) Zur naturschutzfachlichen Situation der Binnendünen in Schleswig-Holstein. Jahresbericht des Landesamtes für Natur und Umwelt des Landes Schleswig-Holstein 2007/ 2008:177-190.

Geologischer Dienst LLUR Schleswig-Holstein (2012) Geologische Übersichtskarte von Schleswig-Holstein 1.250000. Kiel.

Glaser F, Müller H (2003) Wiederfund von Formica (Coptoformica) foreli Bondroit, 1918 und erster sicherer Nachweis von Formica (C.) pressilabris Nylander, 1846 in Österreich (Hymenoptera, Formicidae). Myrmecologische Nachrichten, 5:1-5.

Kutter H (1956) Beiträge zur Biologie palaearktischer Coptoformica (Hym. Form.). Beilage zu Mitteilungen der Schweizerischen Entomologischen Gesellschaft 29 (1):1-18.

Kutter H (1977) Hymenoptera: Formicidae. Schweizerische Entomologische Gesellschaft, Zürich.

Loon AJv (2004) Formicidae - mieren. In: Peeters TMJ et. al (Hg.) De wespen en mieren van Nederland (Hymenoptera: Aculeata). Nationaal Natuurhistorisch Museum Naturalis, Leiden, knnv Uitgeverij, Utrecht, European Invertebrate Survey, Leiden, S. 227-263.

Nielsen MG, Larsen R (2012) Myrer i Danmark. Natur og Museum, Aarhus.

Ødegaard F, Olsen KM, Staverløkk A, Gjershaug JO (2015) Towards a new era for the knowledge of ants (Hymenoptera, Formicidae) in Norway? Nine species new to the country. Nor. J. Entomol. 62:80-99.

Riedel W (1978) Landschaftswandel und gegenwärtige Umweltbeeinflussung im nördlichen Landesteil Schleswig. Schleswiger Druck- und Verlagsgesellschaft, Schleswig

Runge F (1990) Die Pflanzengesellschaften Mitteleuropas. Eine kleine Übersicht. 11. Auflage. Aschendorff Verlag, Münster.

Schultz R, Seifert B (2007) The distribution of the subgenus Coptoformica Müller, 1923 (Hymenoptera: Formicidae) in the Palaearctic Region. Myrmecological News 10:11-18.

Seifert B (2000) A taxonomic revision of the ant subgenus Coptoformica Mueller, 1923 (Hymenoptera, Formicidae). Zoosystema22 (3):517-568.

Seifert B (2007) Die Ameisen Mittel- und Nordeuropas. Lutra Verlagsund Vertriebsgesellschaft, Tauer.

Seifert B (2011) Rote Liste und Gesamtartenliste der Ameisen (Hymenoptera: Formicidae) Deutschlands. In: Binot-Hafke M et. al (Red.) Rote Liste gefährdeter Tiere, Pflanzen und Pilze Deutschlands. Bd. 3 : Wirbellose Tiere (Teil 1). Landwirtschaftsverlag, Münster, S. 469-487.

Seifert B (2018) The Ants of Central and North Europe. Lutra Verlagsund Vertriebsgesellschaft, Tauer.

Sörensen U (1993) Zur Berücksichtigung faunistischer Daten bei Heidepflegemaßnahmen in Schleswig-Holstein. NNA-Berichte6 (3):63-66.

Sörensen U (1996) Untersuchung zur Konstanz und Variabilität der Neststandorte von Coptoformica forsslundi Lohmander (Hymenoptera: Formicidae) im Naturschutzgebiet "Süderlügumer Binnendünen" (Nordfriesland). Gutachten im Auftrag des Umweltamtes Nordfriesland, Husum.

Sörensen U (1999) Coptoformica forsslundi Lohmander, 1949 - eine neue Ameisenart in Deutschland (Hymenoptera: Formicidae). FaunistischÖkologische Mitteilungen 7 (11/12):481-496.

Sörensen U (2004) Ökologie der Kerbameise Coptoformica forsslundi Lohmander, 1949 (Hymenoptera: Formicidae, genus Formica, subgenus 
Coptoformica) an ihrem norddeutschen Vorkommen. Dissertation, Fachbereich Biologie, Universität Hannover, doi: 10.15488/9630.

Sörensen U (2006) Der Einfluss der Hüteschafbeweidung auf gefährdete Ameisenarten (Hymenoptera, Formicidae) im NSG Süderlügumer Binnendünen/ NF. Gutachten auf der Grundlage von Freilanduntersuchungen in den Jahren 2002 bis 2006. Erstellt im Auftrage der unteren Naturschutzbehörde des Kreises Nordfriesland, Husum.

Sörensen U (2017) Myrmecologisches Gutachten zur Vorbereitung von Pflegemaßnahmen im Naturschutzgebiet Süderlügumer Binnendünen/ Nordfriesland im Jahre 2017. Unveröff. Gutachten für die Schleswig-Holsteinischen Landesforsten (AöR).

Sonnenburg H (2005) Die Ameisenfauna (Hymenoptera: Formicidae) Niedersachsens und Bremens. Braunschweiger Naturkundliche Schriften 7 (2):377-441.

Wesenigk-Sturm B (2002) Ein rezentes Vorkommen von Formica (C.) pressilabris im Land Brandenburg. Ameisenschutz aktuell16 (4):97-98.

Woldstedt P, Duphorn K (1974) Norddeutschland und angrenzende Gebiete im Eiszeitalter. 3. Aufl. K.F. Koehler Verlag, Stuttgart.

\section{Open Access}

>> Der Artikel ist unter der Creative-Commons-Lizenz Namensnennung 4.0 International veröffentlicht. Den Vertragstext finden Sie unter: https://creativecommons.org/licenses/by/4.0/deed.de. Bitte beachten Sie, dass einzelne, entsprechend gekennzeichnete Teile des Artikels von der genannten Lizenz ausgenommen sein bzw. anderen urheberrechtlichen Bedingungen unterliegen können. 